\title{
Developments in sample preparation of advanced semiconductor devices from the bulk to nanometer-length scales
}

\author{
Cecile Bonifacio ${ }^{1}$, Clive Downing ${ }^{2}$, Pawel Nowakowski ${ }^{1}$, Richard $\mathrm{Li}^{1}$, Mary Ray ${ }^{1}$ and Paul Fischione ${ }^{1}$ \\ ${ }^{1}$ E.A. Fischione Instruments, Inc., United States, ${ }^{2}$ The Advanced Microscopy Laboratory, CRANN, Trinity \\ College Dublin, Dublin, Ireland, United States
}

Electron microscopy and microanalysis are continuously evolving, which has led to enhanced resolution for transmission and scanning electron microscopes (TEM and SEM) and improved energy resolution and sensitivity for spectrometers and detectors, both of which are capable of faster acquisitions speeds. Sample preparation tools for delayering and site-specific extraction have kept pace with the advancements in these analytical tools. The most common sample preparation technique is by ion milling, which includes broad ion beam (BIB) Ar milling and focused ion beam (FIB) milling using Ga or Xe plasma. All of these tools are used for the preparation of bulk specimens and TEM specimens, but they are also components of analytical investigations, such as failure analysis in the semiconductor industry. Combining both bulk and site-specific preparations can increase throughput and enhance overall quality of specimens for analysis. In this work, we provide an electron microscopy-focused, highthroughput workflow for sample preparation by Ar ion milling - BIB milling for bulk samples and concentrated ion beam (CIB) milling as post-FIB treatment for TEM specimens. The methodology results in samples free from artifacts caused by mechanical preparation damage and ion retention [1] in bulk samples, and amorphization, Ga implantation, and curtaining from Ga FIB milling in TEM specimens [2-5]. Consequently, these specimens are high quality and suitable for atomic resolution electron microscopy imaging and analysis.

Advanced semiconductor devices, such as $20 \mathrm{~nm}$ NAND [solid state drive, IM Flash Technologies], $14 \mathrm{~nm}$ FinFETs [Broadwell M core processor, Intel], and $7 \mathrm{~nm}$ FinFETs [A12 Bionic chip, TSMC] were prepared. The workflow was BIB milling [Model 1061 SEM Mill, Fischione Instruments] to delayer and target a specific layer in a device for SEM characterization (Figure 1) from which TEM specimens were prepared in a FIB system [Scios DualBeam, Thermo Fisher Scientific]. The TEM specimen was subsequently cleaned by CIB milling at high specimen tilts [Model 1080 PicoMill@ TEM specimen preparation system, Fischione Instruments], which resulted in large, electron-transparent areas. TEM characterization by conventional and aberration-corrected imaging and analysis by energy X-ray dispersive spectroscopy (EDS) and electron energy loss spectroscopy (EELS) were performed. Results (Figure 2) demonstrate the effectivity of the specimen preparation technique in removing preparationinduced artifacts from delayering to FIB preparation for aberration corrected imaging (Fig. 2a-b) and in specimen thinning for low $\mathrm{kV}$ EELS analysis (Fig. 2c).

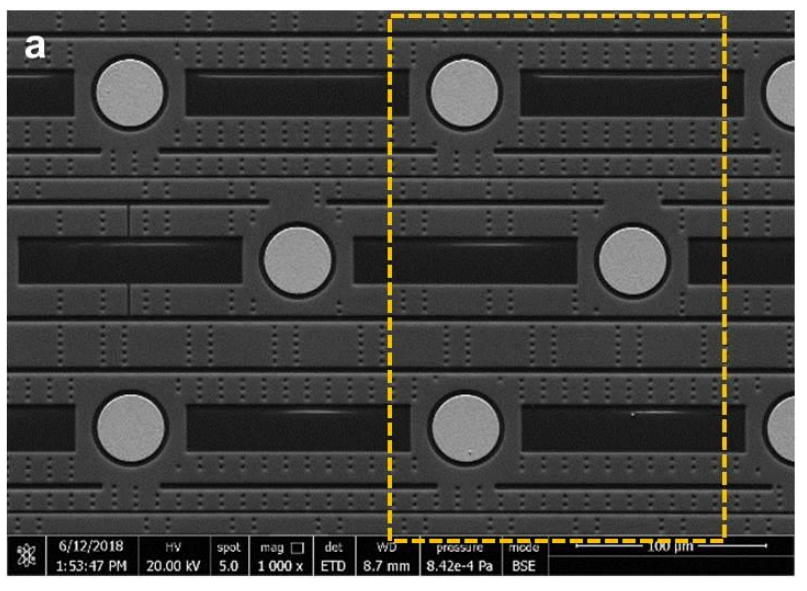

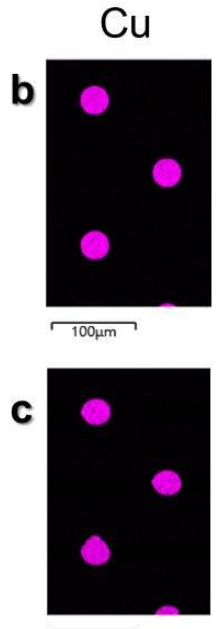

$\longdiv { 1 0 0 \mu \mathrm { m } }$

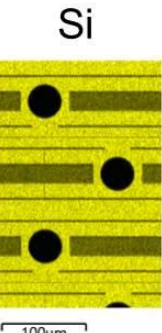

$\longdiv { 1 0 0 \mu \mathrm { m } }$
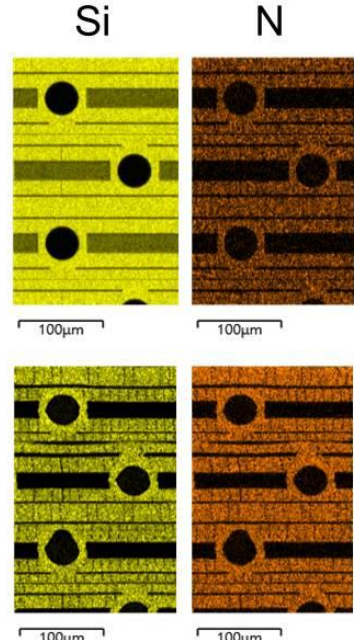

$\longdiv { 1 0 0 \mu \mathrm { m } }$

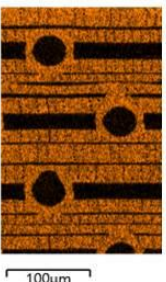

Al

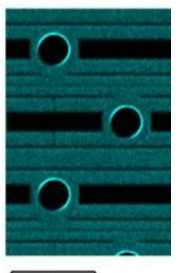

$\longdiv { 1 0 0 \mu \mathrm { m } }$ 
Figure 1. Scanning electron microscope (SEM) image at $20 \mathrm{kV}$ of the delayered $7 \mathrm{~nm}$ finFET device (a). EDS maps at $20 \mathrm{kV}$ (b) and $5 \mathrm{kV}$ (c) indicate $\mathrm{Al}$ layers are below the $\mathrm{SiN}$ and $\mathrm{Cu}$ connectors.
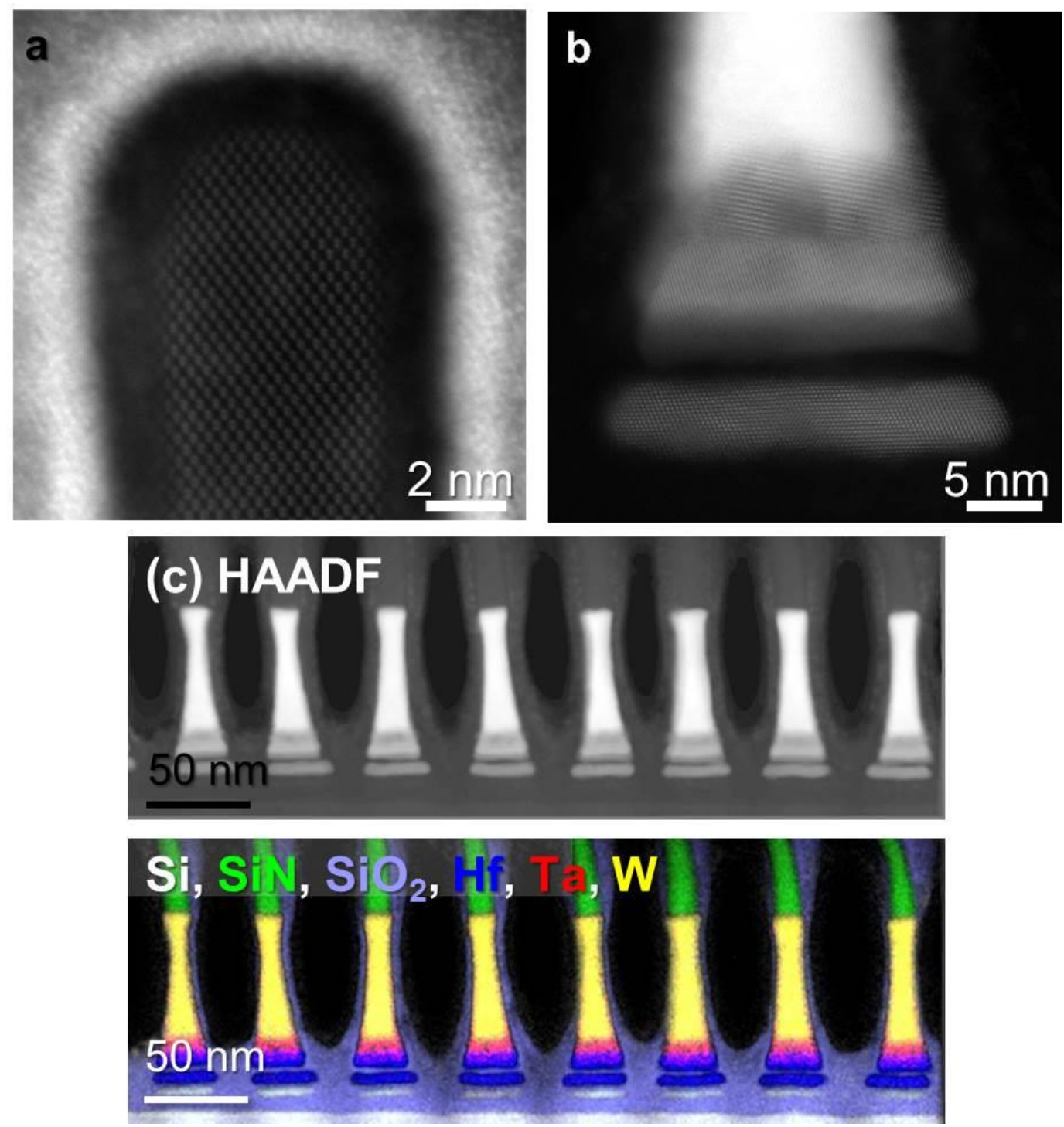

Figure 2. Atomic resolution high-angle annular dark field STEM (HAADF-STEM) images of 7 nm finFET (a) and $20 \mathrm{~nm}$ NAND (b) after Ar ion milling. HAADF and colorized EELS maps at $120 \mathrm{kV}$ on multiple gates of the NAND device (c).

\section{References}

1. Ray, V., Hadjikhani, A., Favata, J., Ahmadi, S., \& Shahbazmohamadi, S. (2017). Further inquiry into Xe primary ion species for circuit edit application. ISTFA 2017: Conference Proceedings from the 43rd International Symposium for Testing and Failure Analysis, 251-255. 
2. Mitome, M. (2012). Ultrathin specimen preparation by a low-energy AR-ion Milling method. Microscopy, 62(2), 321-326. https://doi.org/10.1093/jmicro/dfs073

3. P. E., Williams, R. E., Genç, A., Fraser, H. L., Dunin-Borkowski, R. E., Luysberg, M., Bonifacio, C. S., \& Kovács, A. (2017). A small spot, inert gas, ion Milling process as a complementary technique to focused ion beam specimen preparation. Microscopy and Microanalysis, 23(4), 782793. https://doi.org/10.1017/s1431927617000514

4. Lotnyk, A., Poppitz, D., Ross, U., Gerlach, J., Frost, F., Bernütz, S., Thelander, E., \& Rauschenbach, B. (2015). Focused high- and low-energy ion Milling for TEM specimen preparation. Microelectronics Reliability, 55(9-10), 2119-2125. https://doi.org/10.1016/j.microrel.2015.07.005

5. Montoya, E., Bals, S., Rossell, M. D., Schryvers, D., \& Van Tendeloo, G. (2007). Evaluation of top, angle, and side cleaned FIB samples for TEM analysis. Microscopy Research and Technique, 70(12), 10601071. https://doi.org/10.1002/jemt.20514 